EPJ Web of Conferences 103, 07005 (2015)

DOI: $10.1051 /$ epjconf/ 201510307005

(C) Owned by the authors, published by EDP Sciences, 2015

\title{
Chaotic Regime of Dicke Invertionless Super-Radiance in a High-Q Cavity
}

\author{
I.V. Ryzhov ${ }^{1 a}$, N.A. Vasil'ev ${ }^{1}$, V.A. Malyshev ${ }^{2}$, M.D. Shtager ${ }^{1}$, and I.S. Kosova ${ }^{1}$ \\ ${ }^{1}$ The A.I. Herzen State Pedagogical University of Russia, St. Petersburg \\ ${ }^{2}$ Institute for Advanced Materials, University of Groningen The Netherlands
}

\begin{abstract}
We investigate theoretically different regimes of Dicke superradiance of an ensemble of three-level emitters (L configuration of the operating transitions) placed in a cyclic high-Q cavity. It is shown, that different regimes of three-level Dicke superradiance can be realized: regular dynamics, quasiperiodic and even chaotic behavior.
\end{abstract}

60 years ago, Robert Dicke predicted that an ensemble of two-level systems, initially prepared in their excited states, can radiate coherently due to correlations of emitters by the own emitted field [1]. Later on, this phenomenon attained the name "coherent spontaneous emission - Dicke super-radiance (SR)." SR of an ensemble of two-level systems requires an initial population inversion $[1,2]$. In the case of multilevel emitters, in particular, three-level systems with $\Lambda$-scheme of operating transitions, this limitation can be broken down: the SR can be initiated even if the population of the upper level is smaller than the population of the doublet, i. e, without the total population inversion $[3,4]$. If one prepares the initial state of the doublet in a coherent superposition to which the optical transition from the upper level is forbidden, then the orthogonal superposition, the optical transition to which is allowed, turns out to be unpopulated. Hence, the corresponding optical transition appears to be inverted at any population of the upper state. $\mathrm{LaF}_{3}: \mathrm{Pr}^{3+}$ crystals with a fine structure of the ground state represent an example of such systems $[5,6]$.

We investigate theoretically the SR of an ensemble of three-level $\Lambda$-systems placed in a high-Q cavity within the framework of the semi-classical approach. Results obtained in the present work are: 1. In the case of degenerate doublet, the problem can be mapped into the Duffing oscillator. The analytical solution of the SR developing is obtained. 2. On the basis for the Poincare map, it is demonstrated a feasibility of quasi-periodic and chaotic regimes of the invertionless SR.

\section{References}

1. R.H. Dicke, Phys. Rev. 93. P.99. (1954),

2. M.G.Benedict, A.M. Ermolaev, V.A. Malyshev et al., Super-radiance: Multiatomic Coherent Emission. Bristol and Philadelphia -326 p. (Institute of Physics Publishing, 1996)

3. V.A. Malyshev, I.V. Ryzhov, E.D. Trifonov, A.I. Zaitsev, Las. Phys. 8. P.494 (1998)

4. A.I Zaitsev. et al., JETP. 88, №2. P.278 (1999)

5. A.M. Basharov et al., JETP. 102. №2. P.202 (2006)

6. G.G. Grigoryan et al., Las. Phys. 17. №3. P.511 (2007)

${ }^{a}$ Corresponding author: igoryzhov@yandex.ru

This is an Open Access article distributed under the terms of the Creative Commons Attribution License 4.0, which permits unrestricted use, distribution, and reproduction in any medium, provided the original work is properly cited. 